\title{
La réforme de la formation des enseignants
}

The Reform in Teacher Training

\section{Antoine Delcroix}

\section{OpenEdition}

Journals

Édition électronique

URL : http://journals.openedition.org/rref/926

DOI : $10.4000 /$ rref.926

ISSN : 2494-7830

Éditeur

CRDP de Guadeloupe

\section{Édition imprimée}

Date de publication : 15 décembre 2008

Pagination : 78-81

ISSN : 1962-2864

\section{Référence électronique}

Antoine Delcroix, "La réforme de la formation des enseignants », Recherches et ressources en

éducation et formation [En ligne], 2 | 2008, mis en ligne le 24 avril 2020, consulté le 10 décembre 2020 URL : http://journals.openedition.org/rref/926 ; DOI : https://doi.org/10.4000/rref.926

\section{(c) (7) (8)}

La revue Recherches et ressources en éducation et formation est mise à disposition selon les termes de la Licence Creative Commons Attribution - Pas d'Utilisation Commerciale. 


\section{Notes d'actualité}

\section{La réforme de la formation des enseignants}

Antoine DELCROIX 


\section{Antoine DELCROIX}

Professeur des universités, Directeur du CRREF

IUFM de la Guadeloupe

Contact : antoine.delcroix@iufm.univ-ag.fr

La réforme de la formation des enseignants, voulue par le gouvernement actuel et appuyée sur une argumentation basée sur les recommandations européennes, nous semble pouvoir être analysée de plusieurs points de vue : son inscription dans un processus d'évolution des dispositifs de formation des cadres de la fonction publique, marqué par une élévation du niveau de recrutement initial ; ses conséquences sur les institutions assurant la formation des enseignants ; les conséquences de son application aux Antilles et en Guyane. Nous aborderons moins deux autres aspects : les conséquences sociales de cette réforme, qui fait financer aux étudiants l'intégralité de leur formation ; le risque de voir se multiplier les enseignants contractuels, à coté ou à la place d'enseignants statutaires. Notre propos est plutôt (du moins ici !) de porter un regard sur les enjeux organisationnels et politiques de la réforme. Le point de vue adopté n'est pas celui d'un chercheur, historien ou philosophe de l'éducation, mais celui d'un acteur, engagé à l'heure où ces lignes sont écrites dans la construction des nouveaux dispositifs de formation. J'espère que l'on me pardonnera un éventuel manque de recul ou des prises de position non distanciées.

\section{Une réforme marquant la poursuite d'un processus}

Depuis la mise en place des écoles normales, la formation des enseignants du primaire a toujours possédé une double fonction qui « consistait d'une part dans la transmission d'un capital scolaire sanctionné par l'obtention d'un titre scolaire variable selon les réformes (...). D'autre part elle a toujours consisté dans la transmission d'une formation professionnelle jugée nécessaire à la pratique du métier d'instituteur $»^{I}$. Cependant, le temps consacré dans le curriculum de l'enseignant du primaire à ces deux fonctions a fortement évolué, constamment au détriment de la partie la plus professionnelle de la formation, parallèlement à la montée des exigences de diplôme pour passer le concours. Ainsi, dans la formule en vigueur jusqu'au concours 2009 inclus, le cursus comporte les trois années de licence (grade nécessaire, sauf dérogation pour présenter le concours), typiquement préparées dans une université, une année de préparation au concours, typiquement effectuée en IUFM ${ }^{I I}$ et, après obtention du concours, une année de formation professionnelle, en tant que stagiaire rémunéré affecté en IUFM. Et encore, cette année est un peu écourtée par la nécessité d'organiser la validation et la certification des stagiaires. L'institution a elle-même reconnu l'insuffisance de cette période de formation initiale en ayant prévu des périodes « de formation initiale différée $»^{I I I}$ après la titularisation.

La réforme actuelle, en demandant la double sanction du grade de master et de la réussite à un concours pousse au paroxysme ce processus puisque désormais, la formation initiale universitaire devient moins professionnalisante. Par exemple, à l'heure où ces lignes sont écrites, tout porte à croire qu'aucun stage en responsabilité ne pourra être effectué pendant le master. D'ailleurs, l'adaptation au métier devient du ressort de l'employeur. Elle se fait en tant que fonctionnaire stagiaire, en situation d'exercice à plein temps ${ }^{I V}$. L'intégralité du paragraphe présentant le premier objectif des nouveaux concours mérite d'être repris, tant il est significatif : [Le premier objectif est de] « Mieux distinguer ce qui relève des universités qui ont la responsabilité de la formation initiale de tous les étudiants se destinant au professorat jusqu'au niveau du master et ce qui relève de l'Education nationale qui a la responsabilité du recrutement, de l'adaptation au métier et de la formation continue des professeurs $»^{V}$. On voit que le terme de formation professionnelle est évacué du vocabulaire employé, ce que l'on peut interpréter comme un malaise des concepteurs de la réforme ou bien comme une synthèse difficile entre différents points de vue. A vrai dire, si cela manifestait une reconnaissance implicite du fait que la cohabitation au sein d'un même master d'un double objectif (préparer un concours, assurer une formation professionnelle) était incompatible, on accorderait aux concepteurs cette honnêteté, même dissimulée. Le choix de maintenir un recrutement par voie de concours, par une tradition républicaine formant un des socles de la fonction publique française, pouvait impliquer plusieurs attitudes très acceptables : par exemple, un pré recrutement précoce par voie de concours, suivi d'une formation par une école de type école d'ingénieurs, contractualisée avec l'employeur et placée au sein des universités; ou encore, un concours professionnel, proche d'une certification de compétences, placé à l'issue d'un master réellement professionnel, l'adaptation fine au métier relevant de vraies écoles professionnelles, gérées directement par l'employeur. Pour ces deux exemples, une référence à l'école de la magistrature ${ }^{V I}$ peut venir à l'esprit, mais il est clair que la comparaison tourne court, soyons cyniques, quand les « caisses sont vides » et qu'il s'agit de former d'un coté plusieurs milliers d'enseignants et de l'autre à peine deux cents magistrats par an. Nous, personnels des IUFM, devons nous participer à la mise en place de masters un peu hypocritement professionnels ? Bienvenu au (retour du) « compagnonnage », comme presque seule modalité d'adaptation au métier ! 


\section{ANTOINE DELCROIX | ACTUALITís}

\section{Une réforme portant le risque de démantèlement des IUFM}

L'acte de naissance des IUFM ${ }^{V I I}$ porte en lui les ambiguïtés fondamentales de ces établissements. En premier lieu, en ce qui concerne leur mission en formation initiale des enseignants, ils assurent la préparation d'une partie des candidats aux concours de recrutement et la formation de la totalité des lauréats au métier. Ici se trouve déjà la contradiction entre deux actions, l'une essentiellement académique, l'autre essentiellement professionnelle. Encore ces deux actes étaient-il séparés dans le temps, alors que dans le cadre choisi pour la mastérisation de la formation des enseignants ils deviennent simultanés. En deuxième lieu, la structure administrative choisie, celle d'établissement public administratif, mettait donc à la tête de leur conseil d'administration un représentant de l'état, le recteur, représentant l'employeur, mais inventait dans le même temps un rattachement à une ou plusieurs universités, fortement représentés dans les conseils d'administration des IUFM. Certains pensent que ce choix a été préféré à celui de créer directement une école professionnelle dans les universités, pour éviter une rupture trop grande avec la situation antérieure, qui aurait été mal acceptée par les personnels des écoles normales et les corps d'inspections.

Cette double ambiguité fondatrice allait conduire à l'incertitude sur le sort de ces établissements improbables, pour reprendre le mot d'Alain Bouvier, ancien directeur de l'IUFM de Lyon. Au final, la décision d'intégrer les IUFM aux universités ${ }^{V I I}$ ressembla plus au fait du prince : «je veux qu'il y ait un cadre national et que l'université prenne en charge la formation des maîtres» (François Fillon, l'express, №2793 du 4 au 10 janvier 2005). Au passage, on note l'amorce d'une nouvelle contradiction (corroborée par d'autres propos) entre la volonté d'afficher d'un coté un cadre national pour la formation et de l'autre celle de confier aux universités cette formation, puisque ces derniers établissements, par leur nature juridique et leur tradition historique, jouissent en principe d'une plus grande liberté pédagogique qu'un IUFM.

La réalisation des intégrations des IUFM aux universités procéda d'une de ces bizarreries françaises mélange d'autoritarisme administratif, d'arbitraire et de cuisine locale. Par ailleurs, si la logique d'un IUFM par académie, maintenue par la loi, se comprenait lorsqu'il était l'établissement opérateur de la formation initiale des enseignants, comment la justifier dans le cadre d'école intégrée, alors qu'on reconnaît aux universités une plus large autonomie dans le cadre de la loi LRU ${ }^{I X}$ ? Et donc, si une université le désire au nom de quoi lui interdirait on l'accès au marché de la formation initiale des enseignants, en employant un instant le vocabulaire de l'économie de la connaissance ? Plus qu'une bizarerrie, il s'agit plutôt d'une réforme mal concue et mal gérée (tant par le gouvernement que par les syndicats) dont on laisse aux « petites mains » de l'administration centrale et aux acteurs académiques le soin de se dépêtrer.

L'étape suivante, et peut être ultime, de la brève histoire des IUFM vient de la réforme en cours. On en a déjà montré la probable inefficacité en terme de formation professionnelle. On voit facilement également qu'elle peut être l'occasion d'achever le démantèlement du patrimoine des IUFM : cette fois, les universités ne possédant pas d'IUFM ont demandé explicitement à pouvoir ouvrir des parcours de formation aux métiers de l'enseignement : « Compte tenu de l'autonomie et de la responsabilité des universités, celles-ci, qu'elles soient dotées d'un IUFM ou non, doivent être libres de proposer des parcours adaptés, dans le cadre de leur contrat d'établissement, en fonction des compétences attendues d'un professeur figurant notamment dans un cahier des charges national et dans celui des nouveaux concours $\rrbracket^{X}$. Comment un état qui clame que l'autonomie des universités est le moteur de leur efficacité pourrait-il réguler autoritairement les ouvertures de tels parcours ? Ce qui est donc proposé aux IUFM, c'est le risque d'une mort par dilution : dilution de l'école IUFM au sein des domaines de l'université qu'elle a intégré ; dilution dans le paysage universitaire, suivie d'une reconcentration des masters « enseignements », au moins pour certaines disciplines du second degré, vers quelques universités plus efficaces ou plus riches.

\section{Une réforme aux conditions et aux enjeux particuliers aux Antilles et en Guyane}

Les questions d'éducation ont manifestement une place plus importante aux Antilles et en Guyane qu'en métropole. Pour donner un exemple, la composition du corps enseignant, particulièrement dans l'enseignement primaire, est source de débat. Un enseignant, non créolophone, peut-il être un enseignant efficace dans le contexte diglossique des Antilles ? Un enseignant non au fait du plurilinguisme guyanais et des techniques élémentaires de l'apprentissage du français comme langue étrangère peut-il être efficace en Guyane ? Voilà des questions légitimes que la société peut se poser. (On renvoie, notamment, aux autres contributions de ce numéro pour des éléments pouvant alimenter ce débat.) Si l'on ajoute que les débouchés offerts par l'éducation nationale restent importants au regard de la faiblesse du tissu économique local, on voit que tous les éléments sont réunis pour que l'intégration des IUFM à l'université et la masterisation de la formation des enseignants deviennent des enjeux importants. 
Une structure originale de l'enseignement supérieur

Une seule université, celles des Antilles et de la Guyane, implantée sur trois régions doit intégrer trois IUFM, ceux des académies de Guadeloupe, Guyane et Martinique ${ }^{X I}$. Le contexte de la création de ces trois IUFM, issus de l'éclatement de l'IUFM des Antilles et de la Guyane, était celui d'une forte revendication, particulièrement en Guyane : il s'agissait de donner à chaque pays une certaine maîtrise de la formation des enseignants de son académie au travers de ce levier qu'est l'IUFM ${ }^{X I}$. Les politiques se sont saisis de cet outil en Guyane, moins en Martinique et encore moins en Guadeloupe. De plus l'IUFM, pour la Guyane, était le premier établissement ayant son siège dans la région ce qui était symboliquement fort ${ }^{X I I I}$. L'intégration peut donc y être vécue comme un retour en arrière, une perte d'autonomie. Mais, insistons encore une fois, il faudrait se garder de limiter cette question à celle d'un statut administratif : clairement, les enjeux éducatifs en Guyane sont tels qu'ils justifient des traitements au plus près des réalités ${ }^{X I V}$. Par exemple, les usagers de l'IUFM de Guyane sont, à une écrasante majorité des étudiants et stagiaires se destinant au professorat des écoles ; la proportion de stagiaires en situation ${ }^{X V}$ y est grande par rapport aux deux autres IUFM concernés. A l'inverse, l'IUFM de Martinique, avec une majorité d'étudiants préparant des concours du second degré, possède un profil conforme à celui de la majorité des IUFM métropolitains. L'IUFM de Guadeloupe est entre ces deux situations.

En fait, les profils différents des IUFM locaux, reflétant les besoins différents des pays, des cultures disciplinaires différentes, font de l'intégration et de la réussite de la masterisation de la formation des enseignants un des tests importants de la viabilité de l'université des Antilles et de la Guyane.

\section{Les questions de partage des responsabilités}

Le deuxième angle d'approche peut paraître plus anecdotique par rapport aux implications politiques du premier, puisqu'il concerne les responsabilités qu'auront chacun des acteurs dans ces masters menant aux métiers de l'éducation : IUFM, composantes de l'université, domaines de formation ${ }^{X V I}$. Au premier abord, ce débat est commun à l'ensemble du territoire français, avec une ligne de fracture assez simple : les formations que les universitaires connaissent mal (le professorat des écoles, les conseillers principaux d'éducation et de formation, pour les Antilles et la Guyane) sont laissées aux IUFM. Celles que les universitaires connaissent mieux, par exemple les CAPES, sont revendiquées comme leur propriété. Les motifs les plus avouables sont, en premier, que la préparation aux concours ressortait déjà souvent de l'université et, en second, que les maquettes des nouveaux concours laissent une place importante à ce qui relève des $\mathrm{d}$ isciplines. Le premier argument n'est pas valide aux Antilles et en Guyane, où ces préparations reposaient pour l'essentiel sur des moyens IUFM, en terme de locaux, de personnels administratif et technique, de personnels enseignants. Les IUFM des Antilles et de la Guyane ont formaté (jusqu'à un certain point) leurs ressources humaines pour assurer ces préparations, par exemple en transformant des emplois enseignants de statut second degré en emplois d'enseignants chercheurs. Ils ont, par l'expérience de 18 années et par la formation de formateurs, développé une expertise dans ces domaines. D'ailleurs tant que le terme de master n'était pas prononcé, et qu'il n'y avait donc pas de risque de concurrence par rapport aux masters existants, cette maîtrise par les IUFM des préparations des concours était peu contestée localement et l'implication de l'université restait relativement faible.

\section{Pour une reconnaissance des enjeux réels}

En fait, les questions de structure et de partage de responsabilité évoquées dans les points précédents se rejoignent dans le sens où elles cachent en fait les questions de pouvoir au sein de la structure universitaire. Clairement, il faudra un jour très proche savoir sortir de ce débat confiné pour affirmer que les enjeux sont en fait essentiellement citoyens et politiques, au sens le plus noble des termes : savoir quels enseignants seront, après demain, dans les écoles, collèges et lycées de la Guadeloupe est une question dont la Guadeloupe doit se saisir.

Cette question comporte plusieurs grandes attentes : la capacité à constituer des viviers d'étudiants formés localement, intéressés par les métiers de l'éducation car il serait simplement intolérable de ne pas offrir aux jeunes de ce pays la possibilité d'y exercer ces métiers ; la capacité à conduire des étudiants à réussir des concours restant sélectifs, pour éviter le drame du recours massif aux personnels précaires ; la capacité à leur offrir une formation professionnelle initiale leur permettant une entrée réussie dans le métier; la capacité à inventer, en collaboration avec les académies, une formation continuée, leur permettant d'être les réels « ingénieurs de l'éducation » dont le recrutement au niveau master ne fait que reconnaître la nécessité ; la capacité à conduire ceux qui le souhaitent vers les métiers de la recherche, notamment dans les domaines de l'éducation, pour développer un secteur encore largement en déshérence en Guadeloupe. C'est dans la capacité de l'université et des l'IUFM à construire, puis mettre en oeuvre, sous le regard et avec l'accompagnement des forces sociales, un dispositif répondant à ces attentes, que ce mesurera l'aptitude du système universitaire local à transcender les défauts de la réforme en cours, au profit de la formation des enseignants en Guadeloupe. 


\section{ANTOINE DELCROIX | ACTUALITÉs}

\section{NOTES}

${ }^{I}$ Criblez, Lucien et Hofstetter, Rita (avec Périsset Bagnoud, Danièle) (Ed.). (2000). La formation des enseignant(e) s primaires. Histoire et réformes actuelles. Die Ausbildung von Primarlehrer/innen. Geschichte und aktuelle Reformen. Bern : Peter Lang. 595 p.

${ }^{I I}$ Quoique dans la pratique une faible partie des candidats au concours sont étudiants en IUFM (environ $25 \%$ en Guadeloupe) tandis qu'une majorité des lauréats sont des étudiants, ou anciens étudiants de l'IUFM (environ $60 \%$ en Guadeloupe). Voir également la note 7.

${ }^{I I I} \mathrm{BO}$ du 1er mars 2007. D'un point de vue administratif, il s'agit de formation continue. Rien n'est simple dans le système éducatif français !

${ }^{I V} \mathrm{~A}$ l'heure où ces lignes sont écrites, les syndicats espèrent une inflexion sur ce point, vers un stage comportant des aménagements de service.

${ }^{V}$ Réforme du recrutement et de la formation des enseignants. Point presse - François Perret, 13 octobre 2008. http://www.education.gouv.fr/cid22665/enseignants-reforme-du-recrutement-et-de-la-formation.html, $\quad$ site consulté le 15 octobre 2008.

${ }^{V I}$ On fera le parallèle avec la réforme du concours d'entrée à l'école de la magistrature pour la session 2009 dont les épreuves visent à « permettre de repérer la capacité à acquérir une ou plusieurs des compétences fondamentales » attendues d'un magistrat. Ce n'est guère différent des objectifs de l'épreuve d'entretien des nouveaux concours de recrutement d'enseignants : « L'entretien avec le jury permet de vérifier les connaissances du candidat relatives aux valeurs et aux exigences du service public, au système éducatif et à ses institutions et de manière plus générale à son aptitude à exercer le métier de professeur des écoles. »

http://www.enm.justice.fr/ uses/lib/5784/nouveaux concours acces.pdf. (Site consulté le 15 octobre 2008.)

${ }^{V I I}$ Notamment l'ancien article 721-1 du code de l'éducation, confirmé pour l'aspect relatif aux missions par la loi d'orientation et de programme pour l'avenir de l'école n²005-380 : « La formation des maîtres est assurée par les instituts universitaires de formation des maîtres. Ces instituts accueillent à cette fin des étudiants préparant les concours d'accès aux corps des personnels enseignants et les stagiaires admis à ces concours. » C'est nous qui soulignons les articles. Seul Jack Lang, alors ministre de l'éducation nationale s'était vraiment penché sur cette injustice républicaine qui consistait à n'accueillir dans les IUFM qu'une partie des étudiants préparant les concours, n'arrivant pas au demeurant à mettre en place une réforme en ce sens. On pourra consulter à ce sujet le site de l'agence de mutualisation des universités et établissements.

http://www.amue.fr/presentation/articles/article/jack-lang-reforme-les-iufm/(Site consulté le 15 octobre 2008) VIIIPar la loi citée à la note vii.

${ }^{I X}$ Loi n²007-1199 du 10 août 2007 relative aux libertés et responsabilités des universités. Voir par exemple : http://www.legifrance.gouv.fr/. Sa mise en place coïncida plus ou moins avec les intégrations.

${ }^{x}$ Charte relative aux principes directeurs de la réforme du recrutement et de la formation des enseignants. Conférence des Président d'universités. Séance plénière du jeudi 18 septembre 2008. Voir par exemple :

http://www.cpu.fr/uploads/tx_publications/PositionCPU recrutement enseignants sept08.pdf. (Site consulté le 15 octobre 2008.)

${ }^{X I}$ La situation majoritaire se trouvait être d'académies dans lesquelles plusieurs universités ont leur siège, provoquant un choix d'université intégrante. La situation minoritaire était celle d'académie à université unique, comme Limoges, la Corse, la Réunion.

${ }^{X I I}$ Accessoirement, ces créations mettaient la situation des IUFM en conformité avec la loi, prévoyant un IUFM par académie.

${ }^{X I I L}$ L'université des Antilles et de la Guyane ayant son siège en Guadeloupe, la situation était administrativement la même en Martinique, mais me semble-t-il moins ressentie.

${ }^{X I V}$ Mais le lecteur guadeloupéen pourrait faire remarquer que, par exemple, la situation de Saint Martin nécessite la même attention. Le récent statut de collectivité d'Outre Mer de St Martin vient complexifier le dossier.

${ }^{X V}$ Il s'agit de lauréats d'un concours interne de l'éducation nationale. Ces personnes, pour la Guyane, sont le plus souvent des personnels ayant exercé dans l'enseignement, surtout secondaire avec des statuts précaires. Cette situation témoigne de l'explosion démographique en Guyane et de la difficulté pour le système éducatif à la suivre, ne parlons même pas de l'anticiper. Le lecteur intéressé pourra se reporter aux publications papiers et électroniques de l'INSEE.

http://www.insee.fr/fr/regions/guyane/

${ }^{X V I} \mathrm{La}$ réforme du LMD (licence, master, doctorat) entendait restructurer l'offre de formation des universités autour de grands domaines. Au début laissées relativement libres dans leur choix pour définir ces domaines, les universités se sont vues fortement recommander de suivre pour les masters un découpage en 4 domaines : ALL (Arts, Lettres, Langues), DEG (Droit, Economie, Gestion), SHS (Sciences Humaines et Sociales), STS (Sciences, Technologies, Santé). 